\title{
The human pluripotency gene NANOG/NANOGP8 is expressed in gastric cancer and associated with tumor development
}

\author{
JINGYU ZHANG $^{1 *}$, XIA WANG ${ }^{2,3 *}$, BING CHEN $^{2}$, ZHIFENG XIAO $^{2}$, WENMEI LI $^{1}$, \\ YOUYONG LU ${ }^{1}$ and JIANWU DAI ${ }^{2}$ \\ ${ }^{1}$ Laboratory of Molecular Oncology, Key Laboratory of Carcinogenesis and Translational Research, \\ Peking University School of Oncology, Beijing Cancer Hospital and Institute, Beijing 100142; \\ ${ }^{2}$ Key Laboratory of Molecular Developmental Biology, Institute of Genetics and Developmental Biology; \\ ${ }^{3}$ The Graduate School, Chinese Academy of Sciences, Beijing 100190, P.R. China
}

Received November 23, 2009; Accepted March 11, 2010

DOI: 10.3892/ol_00000081

\begin{abstract}
It is well known that cancer cells exhibit characteristics similar to normal stem cells. The majority of tumors frequently overexpress genes commonly found in embryonic stem cells. To determine whether the pluripotency gene NANOG and its retrogene, NANOGP8, play a role in gastric cancer, we analyzed the NANOG/NANOGP8 expression profile at the mRNA and protein level in primary gastric tumors. Our data demonstrated that overexpression of NANOG/NANOGP8 was consistently detected in primary tumors $(75 \%, 30 / 40)$ compared to adjacent normal tissues $(12.5 \%, 5 / 40)$. Furthermore, NANOG/NANOGP8 was highly expressed in the intestinal metaplasia $(60 \%, 24 / 40)$ and dysplasia tissues $(66.7 \%, 20 / 30)$ compared to normal tissues. These results suggest that NANOG/NANOGP8 exhibits potential as a biomarker and its expression may play an important role in gastric cancer development.
\end{abstract}

\section{Introduction}

Recently, substantial evidence has shown that cancer cells exhibit morphological, biological and phenotypical characteristics similar to normal stem cells. Previous research provided

Correspondence to: Professor Jianwu Dai, Key Laboratory of Molecular Developmental Biology, Institute of Genetics and Developmental Biology, Chinese Academy of Sciences, 3 Nanyitiao, Zhongguancun, Beijing 100190, P.R. China

E-mail: jwdai@genetics.ac.cn

Dr Youyong Lu, Laboratory of Molecular Oncology, Key Laboratory of Carcinogenesis and Translational Research, Peking University School of Oncology, Beijing Cancer Hospital and Institute, Fu-Cheng Road 52, Hai-Dian, Beijing 100142, P.R. China

E-mail: youyonglu@sina.com

*Contributed equally

Key words: NANOG, NANOGP8, gastric cancer, stem cell substantial support for the hypothesis that cancer stem cells contribute to tumorigenesis (1-3). The self-renewal of stem and cancer cells may be regulated by similar signaling pathways (4). Notably, certain poorly differentiated tumors frequently overexpress genes preferentially enriched in embryonic stem (ES) cells, such as NANOG, OCT4 and SOX2 (5-9).

NANOG is a transcription factor that plays a vital role in maintaining the pluripotency and self-renewal capacity of ES cells $(10,11)$. It is well known that NANOG is highly and specifically expressed in ES cells $(10,11)$ and human germ cell tumors (12-14). Recent studies indicated that NANOG is also highly expressed in certain somatic tumors, such as breast $(5,8)$, prostate (6) and cervical cancer (15). Of note is that NANOG has 11 highly homologous pseudogenes (16), some of which are normally expressed in tumors (17-20). Our previous study showed that NANOGP8, a retrogene of NANOG, is expressed in several tumor tissues and cell lines together with NANOG (20). Notably, only one amino acid differs between NANOG and NANOGP8; thus, the two proteins perform similar activities in promoting cell proliferation $(20,21)$. Jeter et al (22) recently reported that in cancer cells, NANOG is derived predominantly from a retrogene locus termed NANOGP8.

Gastric cancer (GC) is one of the most common types of cancer and the second highest cause of cancer-related mortality in the world $(23,24)$. Studies have strongly indicated the existence of cancer stem cells in $\mathrm{GC}(25,26)$. Investigation of $\mathrm{ES}$ cell gene expression in GC tissues may be helpful in understanding the molecular mechanism for the stem cell theory of carcinogenesis. To determine its involvement in GC development, this study analyzed the NANOG/NANOGP8 expression profile in $\mathrm{GC}$ and the correlation between the NANOG/NANOGP8 expression and clinicopathological features.

\section{Materials and methods}

Tissue specimens. GCs, as well as their corresponding adjacent non-neoplastic tissues, intestinal metaplasia and dysplasia tissues were obtained from the tumor bank of the Beijing Cancer Hospital and Institute. Each gastric tissue was collected following patient consent and the approval of the 


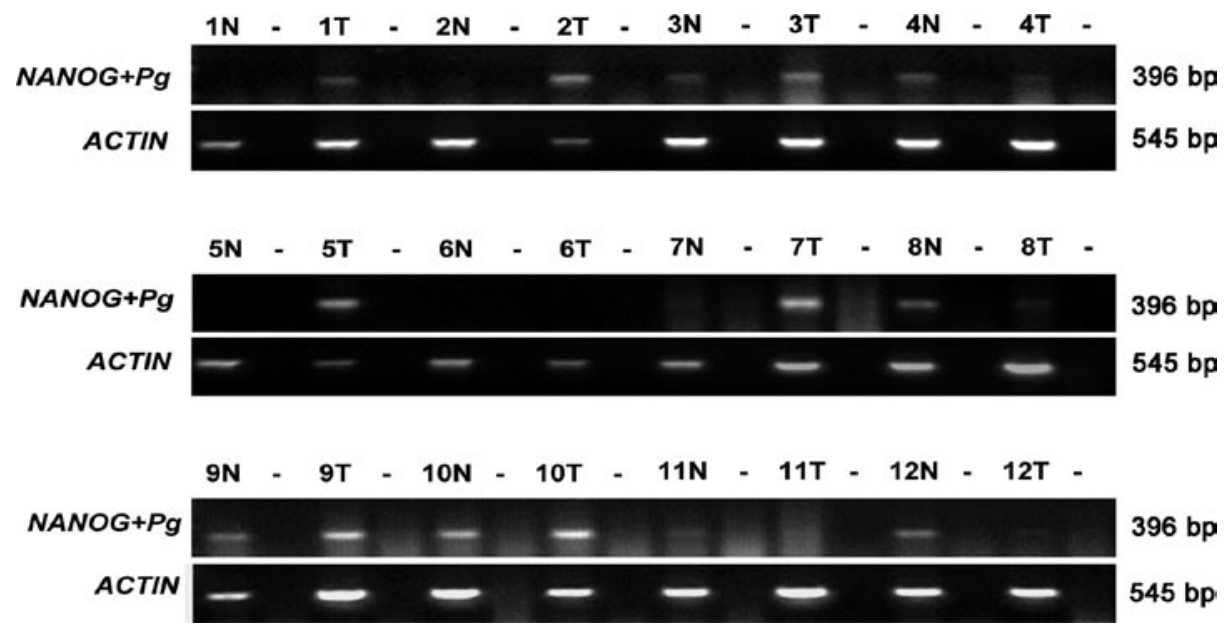

Figure 1. RT-PCR detection of NANOG or its pseudogenes in normal and GC tissues. PCR products indicate the presence of NANOG, NANOGP8 or other pseudogenes of NANOG. Lanes 1-12 represent pairs of specimens from $12 \mathrm{GC}$ patients. Detection of $\beta$-actin was used as a positive control. Products were confirmed by sequencing analysis. T, GC tissue; N, corresponding adjacent normal tissues; -, RT-PCR carried out without reverse transcriptase; Pg, pseudogenes.

ethics committee of the Beijing Cancer Hospital and Institute. The cancer tissues were excised from the central section of the GC. As a control for each GC patient, normal tissue was excised at least $5 \mathrm{~cm}$ from the border of the GC. The specimens were routinely diagnosed by senior pathologists according to pathological biopsy and Lauren's classification. The stage of GC was determined according to the tumornode-metastasis (TNM) classification of the American Joint Committee on Cancer. Follow-up interviews were conducted with patients for at least 5 years or until the patient succumbed to the disease. For tissue microarray analysis, 40 pairs of GC samples, 40 metaplasia tissues and 30 dysplasia tissues were fixed in formalin and embedded in paraffin. For RT-PCR experiments, 12 pairs of samples were frozen in liquid nitrogen within $30 \mathrm{~min}$ after surgery and then stored at $-70^{\circ} \mathrm{C}$ until required.

Total RNA extraction and RT-PCR. Total RNA from gastric samples was extracted using TRIzol reagent (Invitrogen, USA) following procedures described by the manufacturer. To remove any DNA contamination, total RNA was treated with RNase-free DNase I (Takara Bio Inc., Japan). The reverse transcription (RT) reaction was carried out with $2 \mu \mathrm{g}$ total RNA in a $25-\mu 1$ reaction containing M-MLV reverse transcriptase (Promega, USA) using oligo dT primers. An RT reaction was carried out without reverse transcriptase as a negative control. The PCR for NANOG/NANOGP8 was carried out using LATaq ${ }^{\circledR}$ DNA polymerase with GC buffer (Takara Bio Inc.) in a $50-\mu 1$ volume. The NANOG/NANOGP8 primers were: forward, 5'-CCTACCCCAGCCTCTACTCT-3' and reverse, 5'-CGTCTTCAGGTTGCATGTTC-3'. This pair of primers were able to identify not only NANOG/NANOGP8, but also other pseudogenes of NANOG. Amplification of $\beta$-actin was used as a normalizing control. The $\beta$-actin primers were: forward, 5'-CGGGACCTGACTGACTACCTC-3' and reverse, 5'-TCGTCATACTCCTGCTTGCTG-3'. PCR was performed with the following cycling profile: 5 min denaturation at $94^{\circ} \mathrm{C}$ followed by 38 (NANOG/NANOGP8) or 30 cycles ( $\beta$-actin) of
$30 \mathrm{sec}$ at $94^{\circ} \mathrm{C}, 30 \mathrm{sec}$ at $55^{\circ} \mathrm{C}$ and $50 \mathrm{sec}$ at $72^{\circ} \mathrm{C}$, with a final extension step at $72^{\circ} \mathrm{C}$ for $10 \mathrm{~min}$. Products were analyzed by electrophoresis on a $1.2 \%(\mathrm{w} / \mathrm{v})$ agarose gel and the fragments extracted using a gel extraction kit (Omega, USA). Six clones of each specific PCR product were verified by sequencing analysis.

Tissue microarray analysis with immunohistochemistry staining. Tissue microarray blocks of gastric tissues were constructed for immunohistochemistry staining. For each case, five tissue cylinders ( $0.6 \mathrm{~mm}$ diameter, $1 \mathrm{~mm}$ high) were removed from individual GC or adjacent normal tissue. The tissue array blocks were arranged using a puncher (Beecher Instruments, Micro-Array Technologies, USA). Sections $(4 \mu \mathrm{m})$ were obtained from the block and transferred to glass slides. The block contained a representative control of wellmatched cancer and adjacent non-neoplastic tissue.

For immunohistochemical staining, the slides were baked overnight at $60^{\circ} \mathrm{C}$, deparaffinized with xylene and rehydrated in a graded ethanol series. Antigen retrieval was carried out by microwaving tissue in $1 \mathrm{mM}$ EDTA solution ( $\mathrm{pH}$ 8.0). The endogenous peroxidase activity was blocked by incubation in $3 \% \mathrm{H}_{2} \mathrm{O}_{2}$ at room temperature for $10 \mathrm{~min}$. After incubation in $3 \%$ milk to prevent non-specific binding, the slides were immunostained with mouse anti-human NANOG monoclonal antibody (1:100; Abcam, USA) at $4^{\circ} \mathrm{C}$ overnight in a humidified chamber. Slides were washed in phosphate-buffered saline and incubated with a secondary antibody conjugated to peroxidase (Zhongshan Golden Bridge Biotechnology, China). Immunohistochemical staining was performed using a commercial DAB kit (Zhongshan Golden Bridge Biotechnology) which yielded a positive brown signal. Slides were then counterstained with hematoxylin, dehydrated in a graded ethanol series and mounted.

Statistical analysis. The data were analyzed using the Chi-square and Fisher's exact test. The association of NANOG/ NANOGP8 expression with clinicopathological features was 
Table I. Sequencing analysis of RT-PCR products from 12 pairs of specimens taken from GC patients.

\begin{tabular}{|c|c|c|c|c|c|c|}
\hline Case no. & NANOG & NANOGP8 & NANOGP2 & NANOGP4 & NANOGP5 & NANOGP7 \\
\hline \multicolumn{7}{|l|}{1} \\
\hline $\mathrm{T}$ & & & & & & 5 \\
\hline \multicolumn{7}{|l|}{$\mathrm{N}$} \\
\hline \multicolumn{7}{|l|}{2} \\
\hline $\mathrm{T}$ & & 3 & 3 & & & \\
\hline \multicolumn{7}{|l|}{$\mathrm{N}$} \\
\hline \multicolumn{7}{|l|}{3} \\
\hline $\mathrm{T}$ & & & & & 1 & 4 \\
\hline $\mathrm{N}$ & & & 1 & & & 5 \\
\hline \multicolumn{7}{|l|}{4} \\
\hline $\mathrm{T}$ & & & & & 3 & 3 \\
\hline $\mathrm{N}$ & & 1 & 2 & & & 3 \\
\hline \multicolumn{7}{|l|}{5} \\
\hline $\mathrm{T}$ & 2 & 4 & & & & \\
\hline \multicolumn{7}{|l|}{$\mathrm{N}$} \\
\hline \multicolumn{7}{|l|}{6} \\
\hline \multicolumn{7}{|l|}{$\mathrm{T}$} \\
\hline \multicolumn{7}{|l|}{$\mathrm{N}$} \\
\hline \multicolumn{7}{|l|}{7} \\
\hline $\mathrm{T}$ & & 2 & & & & 4 \\
\hline \multicolumn{7}{|l|}{$\mathrm{N}$} \\
\hline \multicolumn{7}{|l|}{8} \\
\hline $\mathrm{T}$ & 1 & & 5 & & & \\
\hline $\mathrm{N}$ & & & 1 & & 3 & 2 \\
\hline \multicolumn{7}{|l|}{9} \\
\hline $\mathrm{T}$ & & 1 & & & 5 & \\
\hline $\mathrm{N}$ & & & 4 & & 1 & 1 \\
\hline \multicolumn{7}{|l|}{10} \\
\hline $\mathrm{T}$ & & & 2 & & & 2 \\
\hline $\mathrm{N}$ & & & 3 & 1 & & 2 \\
\hline \multicolumn{7}{|l|}{11} \\
\hline \multicolumn{7}{|l|}{$\mathrm{T}$} \\
\hline \multicolumn{7}{|l|}{$\mathrm{N}$} \\
\hline \multicolumn{7}{|l|}{12} \\
\hline \multicolumn{7}{|l|}{$\mathrm{T}$} \\
\hline $\mathrm{N}$ & & & 2 & & 2 & 1 \\
\hline
\end{tabular}

T, gastric cancer tissues; $\mathrm{N}$, adjacent normal tissues.

analyzed using SPSS software (version 14). The cumulative survival curve was compared by the log-rank test. For the analyses, $\mathrm{P}<0.05$ was considered to be statistically significant.

\section{Results}

NANOG/NANOGP8 mRNA expression in GC and normal tissues. To compare the expression of NANOG/NANOGP8 between tumor and normal tissues of GC patients, we initally detected mRNA expression using RT-PCR in 12 pairs of specimens. Due to the existence of many NANOG pseudogenes, the primers used in this study were likely able to identify these pseudogenes. Therefore, we examined the PCR products by sequence analysis. As shown in Fig. 1, the presence of NANOG and/or its pseudogene RNA was noted in 10 of the $12 \mathrm{GC}$ cases as compared to normal tissues $(6 / 12)$. The sequencing results obtained from the PCR products indicated that 4 of the $12 \mathrm{GC}$ cases were specific for NANOGP8 and 2 cases contained NANOG, while only 1 of the 12 cases in normal tissues contained the NANOGP8 sequence in the PCR product (Table I). The NANOG pseudogenes, NANOGP2, NANOGP5, NANOGP7 and NANOGP4, 
Table II. Comparison of NANOG/NANOGP8 protein expression between GC and normal matched tissues.

\begin{tabular}{lcccr}
\hline Histology & Total cases & \multicolumn{2}{c}{ NANOG/NANOGP8 } & P-value \\
\cline { 3 - 4 } & & Positive $(\%)$ & Negative (\%) & \\
\hline Tumor & 40 & $30(75.0)$ & $10(25.0)$ & $<0.001$ \\
Normal & 40 & $5(12.5)$ & $35(87.5)$ & \\
\hline
\end{tabular}

Table III. Correlation analysis between NANOG/NANOGP8 protein expression and clinicopathological features.

\begin{tabular}{|c|c|c|c|c|}
\hline \multirow[t]{2}{*}{ Clinicopathological features } & \multirow[t]{2}{*}{ Total cases } & \multicolumn{2}{|c|}{ NANOG/NANOGP8 } & \multirow[t]{2}{*}{ P-value } \\
\hline & & Positive & Negative & \\
\hline Differentiation & & & & 0.589 \\
\hline Well & 13 & 9 & 4 & \\
\hline Poor & 27 & 21 & 6 & \\
\hline $\mathrm{LN}$ metastasis & & & & 0.473 \\
\hline $\mathrm{N}_{0}$ & 12 & 8 & 4 & \\
\hline $\mathrm{N}_{1}$ & 28 & 22 & 6 & \\
\hline Distant metastasis & & & & 1.000 \\
\hline $\mathrm{M}_{0}$ & 36 & 27 & 9 & \\
\hline $\mathrm{M}_{1}$ & 4 & 3 & 1 & \\
\hline Stage & & & & 0.915 \\
\hline $\mathrm{I}, \mathrm{II}$ & 14 & 10 & 4 & \\
\hline III, IV & 26 & 19 & 7 & \\
\hline
\end{tabular}

LN, lymph node.

were also detected in both GC (8/12) and normal tissues $(6 / 12)$ (Table I).

Detection of the NANOG/NANOGP8 protein expression in $G C$ and normal tissues. Of note is that pseudogenes, including NANOG, cannot be translated into functional protein products (27). We attempted to detect NANOG/NANOGP8 protein in GC and normal tissues using immunohistochemical staining. Results indicated that there was an increased NANOG/ NANOGP8 protein expression in GC tissues compared to normal matched tissues (Fig. 2, Table II). Positive NANOG/ NANOGP8 staining was detected in $75 \%$ (30/40) of the GC tissues and only in $12.5 \%(5 / 40)$ of the corresponding adjacent normal tissues $(\mathrm{P}<0.001)$. The majority of NANOG/ NANOGP8 cells were diffusely localized in both the nucleus and cytoplasm (Fig. 2A and B), whereas in other GC tissues, NANOG/NANOGP8 cells were clearly localized in the nucleus (Fig. 2C and D).

Correlation analysis of NANOG/NANOGP8 expression and clinicopathological features. A correlation analysis of NANOG/NANOGP8 expression and the clinicopatho- logical features of GC was conducted. NANOG/NANOGP8 protein expression did not correlate to the following clinicopathological features: GC histological grade (well vs. poor differentiation, $\mathrm{P}=0.589)$, lymph node metastasis $\left(\mathrm{N}_{0}\right.$ vs. $\mathrm{N}_{1}$, $\mathrm{P}=0.473)$, distant metastasis $\left(\mathrm{M}_{0}\right.$ vs. $\left.\mathrm{M}_{1}, \mathrm{P}=1\right)$ or stage (TNM classification, stages I and II vs. stages III and IV, $\mathrm{P}=0.915)$ (Table III). Additionally, the cumulative survival curve indicated no significant difference in the survival time of patients with higher levels vs. those with low levels of NANOG/ NANOGP8 expression ( $\mathrm{P}=0.998$; data not shown).

NANOG/NANOGP8 in the early developmental stages of $G C$. As NANOG/NANOGP8 protein expression in GC did not correlate to the afore-mentioned clinicopathological features, we hypothesized that NANOG/NANOGP8 plays a role in the early developmental stages of gastric carcinogenesis. We were able to detect the NANOG/NANOGP8 protein in the intestinal metaplasia and dysplasia tissues, representative of the early developmental stages of GC $(28,29)$. As shown in Fig. 3, NANOG/NANOGP8 was highly expressed in the intestinal mucosa of the metaplasia tissues $(60 \%, 24 / 40$; Fig. 3A and B). The dysplasia tissues also demonstrated high 

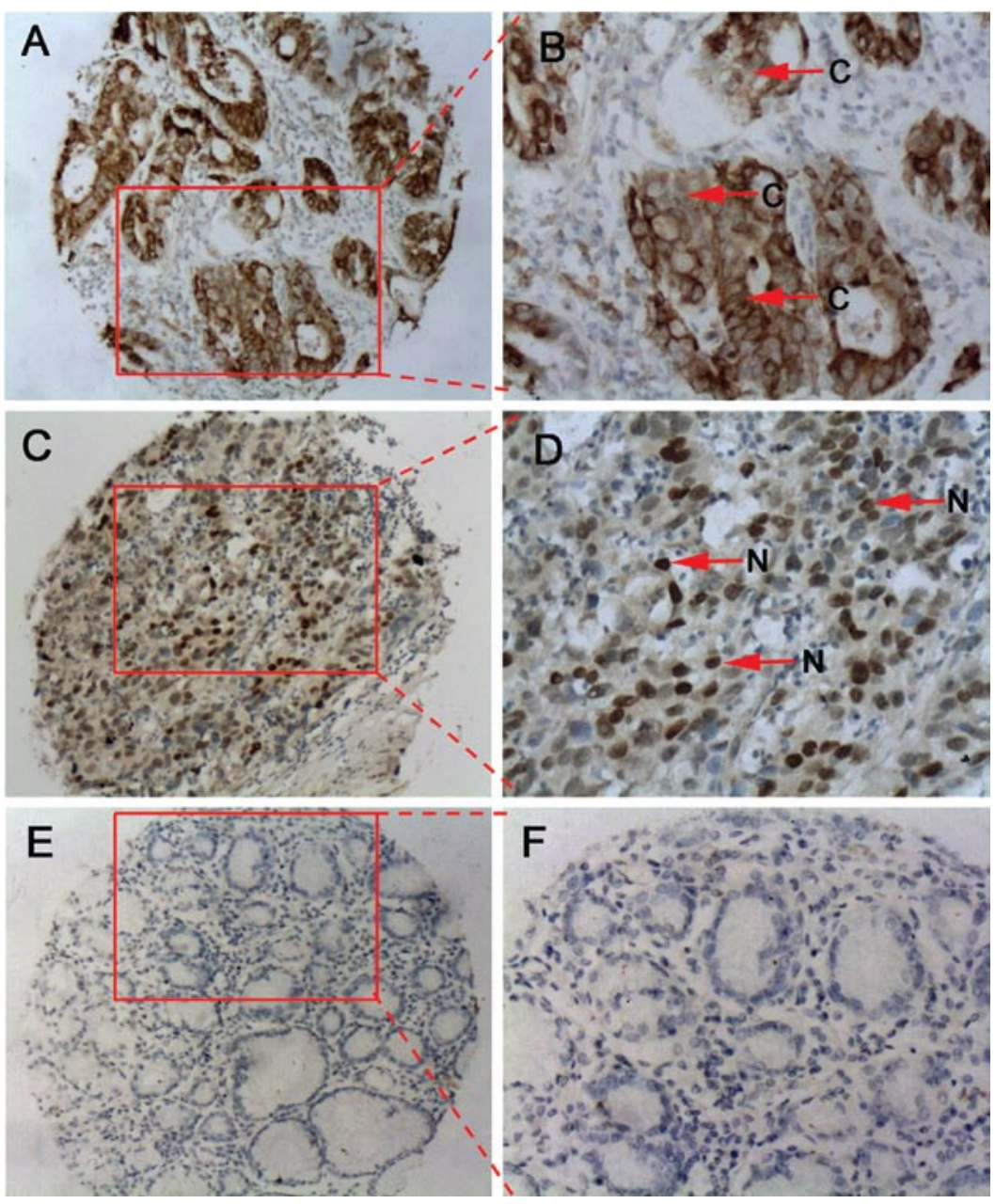

Figure 2. Representative images of immunohistochemical staining for NANOG/NANOGP8 in GC and normal tissues. (A and B) GC tissues showing cytoplasmic localization of NANOG/NANOGP8. (C and D) GC tissues showing the localization of NANOG/NANOGP8 in the nucleus. (E and F) Normal gastric tissues. N, nucleus; C, cytoplasm. A, C and E: magnification, x10; B, D and F: magnification, x20.
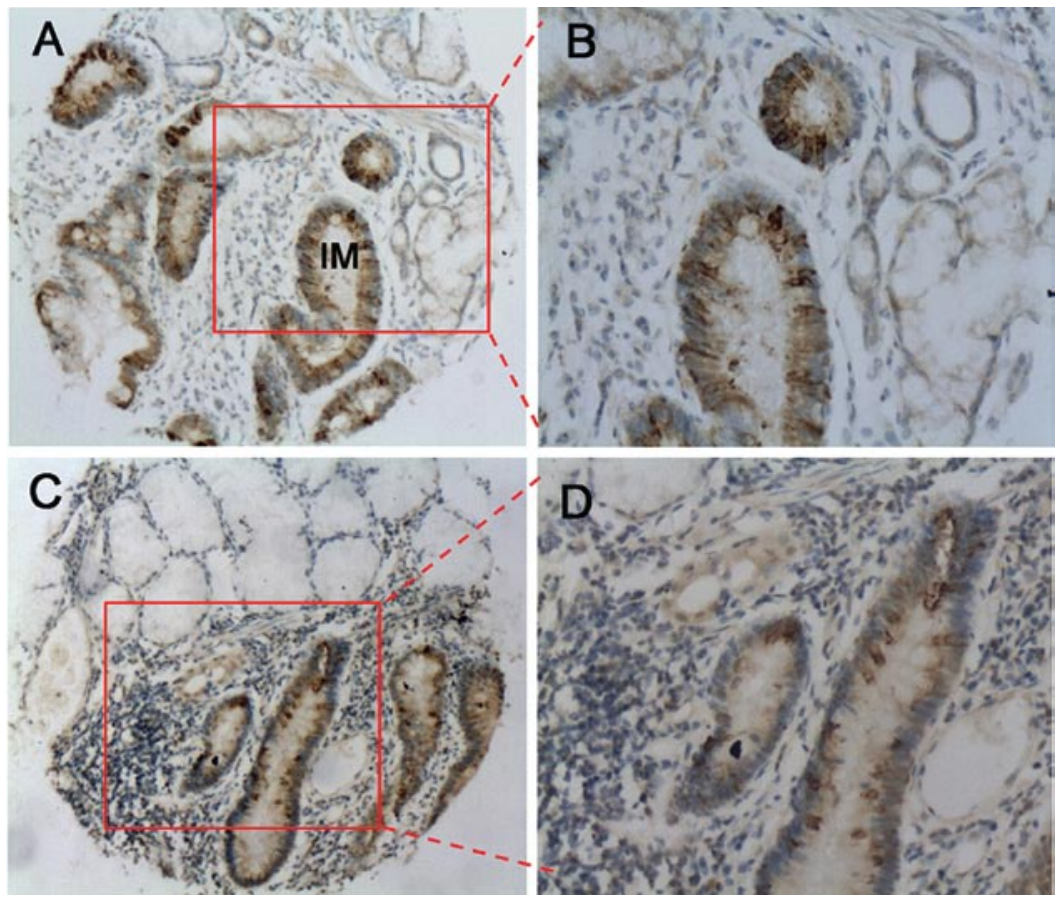

Figure 3. NANOG/NANOGP8 protein expression in the early developmental stages of GC. (A and B) Intestinal metaplasia and (C and D) dysplasia showing the expression of NANOG/NANOGP8, respectively. IM, intestinal mucosa. A and C: magnification, x10; B and D: magnification, x20. 
levels of NANOG/NANOGP8 expression (66.7\%, 20/30), whereas adjacent normal gastric tissues exhibited no expression (Fig. 3C and D).

\section{Discussion}

Of note is that malignant cells have a close relationship to stem cells. It has been postulated that the self-renewal pathways in stem cells perform a similar role in cancer cells $(4,5)$. This study attempted to provide evidence that the ES cell selfrenewal gene, NANOG, is also highly expressed in cancer cells. Since NANOGP8 showed similar activities to NANOG in promoting cell proliferation $(20,21)$, we postulated that NANOG and NANOGP8 play similar roles in maintaining the high proliferative capacity of cancer cells. The mechanism by which NANOG and NANOGP8 expression is regulated may be distinctly different. However, the effects of NANOG and NANOGP8 in carcinogenesis are the same. Furthermore, it is difficult to distinguish the protein products of NANOG and NANOGP8 by immunohistochemistry due to only one amino acid difference between them. Therefore, this study analyzed the expression of NANOG and NANOGP8 together.

We detected an increase in the level of mRNA transcripts of NANOG, NANOGP8 and several NANOG pseudogenes in GC vs. normal tissues. From the 10 RT-PCR-positive cases in GC tissues, six showed an expression of NANOG/NANOGP8 at the mRNA level. The protein expression of NANOG/ NANOGP8 in GC tissues was found to be significantly higher $(75 \%)$ than that of normal tissues $(12.5 \%)$, supporting our hypothesis that the gene NANOG is highly expressed in GC.

It is well known that the transcription factor NANOG, is localized to the nucleus $(21,30)$. The same applies to NANOGP8 (20). Following the exclusion of false-positive staining, we found that in certain cancer specimens, the localization of NANOG/NANOGP8 is not restricted to the nucleus but can also be observed in the cytoplasm. These results are similar to those found in breast carcinoma samples (8) and malignant cervical epithelial cells (15). It provides a hint that NANOG/NANOGP8 plays a more complex role(s) in cancer than anticipated. The regulation mechanism for localization is currently unknown and further study is required.

Investigation into the activities of these genes and their association with cancer may result in a better understanding of the biological characteristics of carcinoma. For the first time, we have proven that no correlation exists between NANOG/ NANOGP8 protein expression and the differentiation, lymph node metastasis, distant metastasis, stage or survival of GC patients. Our results suggest that NANOG/NANOGP8 expression is not related to the prognosis of GC. With respect to the theory that cancer stem cells account for carcinogenesis (1-3), we presume that NANOG/NANOGP8 expression may relate to the initial stages of GC. Intestinal-type gastric tumors are usually well-characterized by sequential developmental stages that include chronic gastritis, atrophy, intestinal metaplasia, dysplasia and carcinoma $(28,29)$. We identified the expression of NANOG/NANOGP8 in the early developmental stages of GCs, during intestinal metaplasia and dysplasia, but not in the adjacent normal tissues. This suggests a potential role for NANOG/NANOGP8 in the early developmental stages of gastric carcinogenesis.
Jeter et al (22) recently reported that NANOG and/or NANOGP8 regulates prostate tumor development. These authors found that the ectopic expression of patient tumorderived NANOGP8 in the K14 cellular compartment of transgenic mice disrupts tissue homeostasis associated with hyperplasia, dysplasia and abnormal differentiation. The results obtained by Jeter et al (22) confirm and significantly extend our findings in GC.

In conclusion, we detected a significantly increased expression of NANOG/NANOGP8 in GC compared to normal tissues. Our results suggest that NANOG/NANOGP8 is not related to the prognosis of GC, but to the early developmental stages of gastric carcinogenesis. Further studies and an analysis of the NANOG/NANOGP8 expression would provide a better understanding of the biological characteristics of GC.

\section{Acknowledgements}

This work was supported by grants from the National Natural Science Foundation of China (30600304), the Ministry of Science and Technology of China (2006CB943601; 2004CB518708; 2006AA02A402) and the Beijing Municipal Science and Technology Commission (D0905001040631). We thank the tissue bank of the Beijing Cancer Hospital and Institute for the gastric tissues.

\section{References}

1. Burkert J, Wright NA and Alison MR: Stem cells and cancer: an intimate relationship. J Pathol 209: 287-297, 2006.

2. Li L and Neaves WB: Normal stem cells and cancer stem cells: the niche matters. Cancer Res 66: 4553-4557, 2006.

3. Marx J: Cancer research. Mutant stem cells may seed cancer. Science 301: 1308-1310, 2003.

4. Reya T, Morrison SJ, Clarke MF and Weissman IL: Stem cells, cancer and cancer stem cells. Nature 414: 105-111, 2001.

5. Ben-Porath I, Thomson MW, Carey VJ, Ge R, Bell GW, Regev A and Weinberg RA: An embryonic stem cell-like gene expression signature in poorly differentiated aggressive human tumors. Nat Genet 40: 499-507, 2008.

6. Gu G, Yuan J, Wills M and Kasper S: Prostate cancer cells with stem cell characteristics reconstitute the original human tumor in vivo. Cancer Res 67: 4807-4815, 2007.

7. Gibbs CP, Kukekov VG, Reith JD, Tchigrinova O, Suslov ON, Scott EW, Ghivizzani SC, Ignatova TN and Steindler DA: Stem-like cells in bone sarcomas: implications for tumorigenesis. Neoplasia 7: 967-976, 2005.

8. Ezeh UI, Turek PJ, Reijo RA and Clark AT: Human embryonic stem cell genes OCT4, NANOG, STELLAR and GDF3 are expressed in both seminoma and breast carcinoma. Cancer 104: 2255-2265, 2005

9. Monk M and Holding C: Human embryonic genes re-expressed in cancer cells. Oncogene 20: 8085-8091, 2001.

10. Chambers I, Colby D, Robertson M, Nichols J, Lee S, Tweedie S and Smith A: Functional expression cloning of Nanog, a pluripotency sustaining factor in embryonic stem cells. Cell 113: 643-655, 2003.

11. Mitsui K, Tokuzawa $\mathrm{Y}$, Itoh H, Segawa K, Murakami M, Takahashi K, Maruyama M, Maeda M and Yamanaka S: The homeoprotein Nanog is required for maintenance of pluripotency in mouse epiblast and ES cells. Cell 113: 631-642, 2003.

12. Hart AH, Hartley L, Parker K, Ibrahim M, Looijenga LH, Pauchnik M, Chow CW and Robb L: The pluripotency homeobox gene NANOG is expressed in human germ cell tumors. Cancer 104: 2092-2098, 2005.

13. Hoei-Hansen CE, Almstrup K, Nielsen JE, Brask Sonne S, Graem N, Skakkebaek NE, Leffers H and Rajpert-De Meyts E: Stem cell pluripotency factor NANOG is expressed in human fetal gonocytes, testicular carcinoma in situ and germ cell tumours. Histopathology 47: 48-56, 2005. 
14. Clark AT, Rodriguez RT, Bodnar MS, Abeyta MJ, Cedars MI, Turek PJ, Firpo MT and Reijo Pera RA: Human STELLAR, NANOG and GDF3 genes are expressed in pluripotent cells and map to chromosome 12p13, a hotspot for teratocarcinoma. Stem Cells 22: 169-179, 2004.

15. Ye F, Zhou C, Cheng Q, Shen J and Chen H: Stem-cell-abundant proteins Nanog, Nucleostemin and Musashil are highly expressed in malignant cervical epithelial cells. BMC Cancer 8 : $108,2008$.

16. Booth HA and Holland PW: Eleven daughters of NANOG. Genomics 84: 229-238, 2004.

17. Kandouz M, Bier A, Carystinos GD, Alaoui-Jamali MA and Batist G: Connexin43 pseudogene is expressed in tumor cells and inhibits growth. Oncogene 23: 4763-4770, 2004.

18. Fujii GH, Morimoto AM, Berson AE and Bolen JB: Transcriptional analysis of the PTEN/MMAC1 pseudogene, psiPTEN. Oncogene 18: 1765-1769, 1999.

19. Suo G, Han J, Wang X, Zhang J, Zhao Y, Zhao Y and Dai J: Oct 4 pseudogenes are transcribed in cancers. BBRC 337: 1047-1051, 2005.

20. Zhang J, Wang X, Li M, Han J, Chen B, Wang B and Dai J: NANOGP8 is a retrogene expressed in cancers. FEBS $\mathrm{J} 273$ : 1723-1730, 2006

21. Zhang J, Wang X, Chen B, Suo G, Zhao Y, Duan Z and Dai J: Expression of Nanog gene promotes NIH3T3 cell proliferation. BBRC 338: 1098-1102, 2005.
22. Jeter CR, Badeaux M, Choy G, Chandra D, Patrawala L, Liu C, Calhoun-Davis T, Zaehres H, Daley GQ and Tang DG: Functional evidence that the self-renewal gene NANOG regulates human tumor development. Stem Cells 27: 993-1005, 2009.

23. Parkin DM, Bray F, Ferlay J and Pisani P: Global cancer statistics, 2002. CA Cancer J Clin 55: 74-108, 2005.

24. Fox JG and Wang TC: Inflammation, atrophy and gastric cancer. J Clin Invest 117: 60-69, 2007.

25. Takaishi S, Okumura T and Wang TC: Gastric cancer stem cells J Clin Oncol 26: 2876-2882, 2008.

26. Schier S and Wright NA: Stem cell relationships and the origin of gastrointestinal cancer. Oncology 1: 9-13, 2005.

27. Balakirev ES and Ayala FJ: Pseudogenes: are they 'junk' or functional DNA? Ann Rev Genet 37: 123-151, 2003.

28. Correa P: A human model of gastric carcinogenesis. Cancer Res 48: 3554-3560, 1988.

29. Correa P: Human gastric carcinogenesis: a multistep and multifactorial process - first American Cancer Society award lecture on cancer epidemiology and prevention. Cancer Res 52: 6735-6740, 1992.

30. Do HJ, Lim HY, Kim JH, Song H, Chung HM and Kim JH: An intact homeobox domain is required for complete nuclear localization of human Nanog. BBRC 353: 770-775, 2007. 